\title{
Common Radio Resource Management for Access Selection in Multi-Access Networks
}

\author{
Fanchun Jin ${ }^{1}$, Hyeong-Ah Choi ${ }^{1}$, Jae-Hoon Kim ${ }^{2}$, JungKyo Sohn ${ }^{3}$, and Hyeong In $\mathrm{Choi}^{3}$ \\ ${ }^{1}$ Department of Computer Science, George Washington University, Washington, DC \\ ${ }^{2}$ Technology Strategic Group, SK Telecom, Seoul, Korea \\ ${ }^{3}$ Department of Mathematics, Seoul National University, Seoul, Korea
}

\begin{abstract}
In this paper, we address the problem of access selection in multi-access networks. As admission and handover process durations are in the order of seconds and decentralized decisions are made over multiple cells spread over the network, such decisions have to be made quickly and in a distributed manner while keeping the network stable and its load balanced. We propose a utility-based approach to access selection. In this model, policies governing the network operations are embodied in the design of marginal utility functions and decisions are made using minimal computations. Developed algorithms are then evaluated using a Java-based multi-access simulator called MANSim that we developed.
\end{abstract}

\section{INTRODUCTION}

Next-generation wireless networks are expected to provide reliable, high-bandwidth, on-demand services with performance guarantees to a variety of users with diverse traffic characteristics and hardware capabilities. Users may freely move across all network providers and regions changing computing and communication devices while maintaining the continuity of their applications. In such a network referred to as a multi-access network, a mobile station (MS) is equipped with a hardware compatible to multiple radio access technologies (RATs). One of the key management issues in a multi-access network is the access selection for call admissions and and vertical handovers (handovers to cells using different RATs). Significant research has focussed on developing access selection schemes that incorporate network-, mobile-, and service-specific information.

In this paper, we present a utility-based access selection framework. Two implementation options are discussed, and the performance of each option is analyzed under a specific network operation model. The rest of the paper is organized as follows. In Section II, world-wide on-going projects in multi-access networks are briefly introduced with a relevant literature survey. In Section III, a system model and network operational policy rules are presented to demonstrate the validity of our proposed scheme. The design of marginal utility functions incorporating the given operational policy rules are then presented in Section IV. Using the utility values computed by the marginal utility functions, two implementation options are proposed in Section V. The performance of each implementation is analyzed using a simulator called the MANSim (Multi-Access Network Simulator) that we developed, and results are presented in
Section VI. Concluding remarks and the direction of future works are given in Section VII.

\section{RELATED WORK}

In a multi-access heterogeneous network, each individual network separately operates and provides services to users. Mobile stations are equipped with a hardware supporting multiple RATs, and an individual RAT is selected to provide service to a user. Thus, the main concept of the multi-access network is not to combine and control all possible networks together, instead, to provide a flexible and open architecture for a large variety of different mobile wireless networks, applications and services with different QoS demands, as well as different protocol stacks.

There are many research projects toward beyond third generation (B3G) networks aiming at multi-access heterogeneous networks such as the European Union 6th Framework Program (FP6), the FuTURE in China, the Future Wireless World launched by DoD in North America, the NGMC Forum in South Korea, and the Mobile IT Forum (mITF) in Japan. Among these, the Ambient Networks project, a part of the FP6, is in the most advanced stage. It has developed a generic multi-radio access architecture [1] and a conceptual framework for access selection and resource allocation by joint radio resource management across different RATs [2].

There are two architecture models commonly considered for inter-networking: IP level integration and radio access level integration. In the IP-based approach, the network remains independent of each other and potentially belongs to different administrative domains. In the radio access level integration, the control protocols of one network can be reused within the other network, and data traffic can be routed via the other's core network. The former is called loosely-couple while the latter is called tightly-coupled. The loosely-couples architectures are discussed in [3], [4], [5], and the tightly-coupled architectures are discussed in [6], [7], [8], [9] An architecture for UMTS and 802.16 integration is presented in [10]. [11] presents a vertical handoff system that minimizes the vertical handoff latency for an individual user while keeping bandwidth and power overheads as low as possible. In [12], [13], the problem of allocating user connections to a set of base stations is considered with the objectives of minimizing power consumption of mobile stations and maximizing admitted traffic flows while 
following access preferences of users using bin packing algorithm based heuristics. The issue of load balancing is not addressed in this paper. Another approach to access selected using heuristic bin-packing algorithm is presented The problem of access selection between wireless LAN and HSDPA/Long Term 3G Evolved networks is addressed in [14], and a simple heuristic algorithm is presented. Most of existing works focus on the architecture and protocols, and consider minimizing handover delays. In [15], the authors consider general resource management issues in heterogeneous networks, and discuss several solutions. However, the utility-based access selection scheme proposed in this paper is different from the existing ones in which utility functions are designed to integrate the given operational policy rules and the loads are balanced across network cells to improve the overall throughput.

\section{SySTEM MODEL}

\section{A. Architecure}

Our inter-networking model assumes a tightly-coupled architecture in which a common radio resource management (CRRM) unit is introduced to connect RNCs of UMTS networks (e.g., WCDMA, HSDPA) and MSC of CDMA networks (e.g., CDMA1x, EvDO). The CRRM collects radio information from each individual network, processes collected information, and distribute results necessary for resource management to each network. The CRRM or each mobile station then makes a final suggestion (e.g., which mobile stations should handover to which network cells). In either case, the actual admission control or vertical handover decision is made by the corresponding LRRM using its own algorithm.

- The CDMA1x and EvDO operate on different spectrum bands while the WCDMA and HSDPA share the same spectrum band.

- Two types of the WCDMA system exist. One is operating alone called WCDMA-alone system. The other is sharing its spectrum with HSDPA called WCDMA-HSDPA system, and a fixed amount of the total power is allocated for WCDMA and HSDPA to share.

\section{B. Operational Policy}

The following operational policy rules are assumed.

- Voice traffic is served by CDMA1x system, WCDMA-only system, or non-HSDPA part of the WCDMA-HSDPA system.

- Data traffic is served by EvDO system or HSDPA part of the WCDMA-HSDPA system.

- Voice traffic has always higher priority than data traffic. Hence, a new voice traffic may be admitted to a non-HSDPA part of a WCDMA-HSDPA cell by dropping or redirecting existing data traffic in its HSDPA part.

- The CDMA1x or WCDMA-alone system has higher priority over WCDMA-HSDPA system for voice traffics.

- The EvDO system has higher priority over WCDMA-HSDPA system for data traffics.

\section{Marginal Utility FunCtion}

For a cell $v$ serving voice calls, its load $x_{v}$ (or capacity $c_{v}$ ) is defined as the number of voice calls currently served (or the maximum number of voice calls that can be served) by $v$. For a cell $d$ serving data traffic, its load $x_{d}$ (or capacity $c_{d}$ ) is defined as the total amount of bandwidth provided by $d$ to mobile stations currently in the cell (or the maximum bandwidth that $d$ can provide). For a WCDMA-HSDPA cell that serve both voice and data traffics, the voice capacity is defined as the maximum number of voice calls that can be served using all the power allocated to the system, and the capacity of data traffic is defined as the maximum amount of bandwidth that the system can provide using all the power allocated to it.

Let $\alpha$ be a real number in $0<\alpha<1$. A marginal cost function for each cell, for voice or data, with a certain RAT is then defined as follows.

- For CDMA1x,

$$
f_{\text {voice }}\left(x_{v}\right)=\begin{array}{cc}
\left(x_{v} / c_{v}\right)^{2} & \text { if } x_{v} / c_{v}<\alpha \\
\left(x_{v} / c_{v}\right)^{2}+1 & \text { otherwise }
\end{array}
$$

- For WCDMA-alone,

$$
f_{\text {voice }}\left(x_{v}\right)=\begin{array}{cc}
\left(x_{v} / c_{v}\right)^{2} & \text { if } x_{v} / c_{v}<\alpha \\
\left(x_{v} / c_{v}\right)^{2}+1 & \text { otherwise }
\end{array}
$$

- For EvDO,

$$
f_{\text {data }}\left(x_{d}\right)=\left(x_{d} / c_{d}\right)^{2}+1
$$

- For WCDMA/HSDPA,

$$
\begin{gathered}
f_{\text {data }}\left(x_{d}\right)=\left(x_{d} / c_{d}\right)^{2} /\left(1-x_{v} / c_{v}\right)^{2}+1 \\
f_{\text {voice }}\left(x_{v}\right)=\left(x_{v} / c_{v}\right)^{2}+1
\end{gathered}
$$

To understand the idea behind the design of these functions, let's first consider voice traffics. The utility value of a CDMA1x (or WCDMA-alone) cell is lower than the utility value of non-HSDPA part of a WCDMA/HSDPA cell whose $x_{v} / c_{v}$ value is same as CDMA1x cell's (or WCDMA cell's) as long as the ratio $x_{v} / c_{v}$ is less than $\alpha$. Hence, CDMA1x or WCDMA-alone cells will have higher priority over nonHSDPA part of WCDMA/HSDPA cells in serving voice traffics. For data traffics, an EvDO cell has lower utility value than a WCDMA/HSDPA serving voice traffic when both cells have the same ratio $x_{d} / c_{d}$. Hence, the EvDO cell has higher priority over the WCDMA/HSDPA cell. Finally, it is noted that the third operational policy rule is also satisfied from the functions defined for WCDMA-HSDPA cells.

Computing the optimal value of $\alpha$ requires precise network modeling and in-depth analysis, and we simply assume $\alpha=0.9$ in our simulations discussed later.

\section{Algorithms}

Each base station periodically updates its utility values and reports them to the CRRM.

\section{A. Network-Based Decision}

When a connection request arrives from a mobile station, the CRRM selects a base station with the minimum utility value, and direct the mobile station to connect to the selected base station. In this approach, each mobile station must periodically report to the CRRM a list of cells (of any RAT type) to which it has enough signal strength to be connected. In this approach, the CRRM has high computation load. The details are as follows. 
1. Every base station reports its load information to CRRM, and CRRM computes utility value for each cell. 2. Let $C(v)$ and $C(d)$, respectively, denote the set of all cells that can accommodate vice and dita tres.

3. While $C(v)$ is not empty, do the following:

(a) Find two neighboring cells, $i$ and $j$, in $C(v)$ with the largest difference of utility values.

(b) Arrange handovers between $i$ and $j$ until they both have the same utility value or

no more handover is possible.

4. While $C(d)$ is not empty, do the followin

(a) Find two neighboring cells, $i$ and $j$, in $C(d)$ with the largest difference of utility values.

(b) Arrange handovers between $i$ and $j$ until they both have the same utility value or no more handover is possible.
Delete $i$ and $j$ from $C(d)$.

1) MS-Based Decision: In this approach, the CRRM guides mobile stations to make final decisions in which the CRRM does not have information on which cells may be accessible by which mobile station (i.e., mobile stations do not report the signal strengths of their accessible base stations to the CRRM). Each base station maintains geographic information of its neighbor cells as specified in the MIH protocol [16]. Suppose MS $m_{j}$ is connected to BS $b_{i}$ whose neighbor set is denoted by $S_{i}$. Note that $m_{j}$ may or may not be able to connect to all base stations in $S_{i}$, but the set of base stations to which the $m_{j}$ can connect must be a subset $S_{i}^{\prime}$ of $S_{i}$. The network (or the CRRM) then delivers the utility value of each cell in the neighbor list $S_{i}$ of $b_{i}$, and $b_{i}$ informs the utility value of each cell in $S_{i}$ to $m_{j} . m_{j}$ then make a final decision using the information on signal strength to each base station in $S_{i}$. More precisely, $m_{j}$ select a cell with the lowest utility value from $S_{i}^{\prime}$.

\section{PERFORMANCE ANALYSIS}

The performance of our CRRM is analyzed using a Javabased system level simulator called MANSim (Multi Access Network Simulator) that we developed. In our simulation, the network consists of the following cells: four CDMA1x cells, four EvDO cells, and two WCDMA-HSDPA. The CDMA1x cells and EvDO cells both cover the entire simulation area. The WCDMA-HSDPA cells each overlapping with at least one other WCDMA-HSDPA cell are deployed in a certain simulation area. The cell capacity of each cell type is defined as follows: 80 voice traffics (equivalently about $1.15 \mathrm{Mbps}$ ) can be accommodated in a single CDMA1x cell; $1.2 \mathrm{Mbps}$ in an EvDO cell; 240 voice traffics (equivalently, about $3.45 \mathrm{Mbps}$ ) in a WCDMA cell when no HSDPA data traffic is served; and $3.6 \mathrm{Mbps}$ in a HSDPA cell when no voice traffic is served. Each mobile station generates one of the five service types of traffic specified in Table I.

\begin{tabular}{|c|c|c|c|}
\hline Service Type & $\begin{array}{c}\text { Bandwidth } \\
\text { Requirement (kbps) }\end{array}$ & $\begin{array}{c}\text { Session } \\
\text { Duration (sec) }\end{array}$ & $\begin{array}{c}\text { Session Inter- } \\
\text { Arrival Time(sec) }\end{array}$ \\
\hline WAP & 9.6 & Weibull $(110,0.54)$ & Exponential (6) \\
\hline VOD Streaming & 64 & Lognormal $(3.0997,0.8951)$ & Exponential (6) \\
\hline VOD Downloading & 48 & Exponential (300) & Exponential (7) \\
\hline Video Conferencing & 64 & Lognormal(6.7, 0.5) & Exponential (7) \\
\hline Voice & 14.4 & Exponential (360) & Exponential (5) \\
\hline
\end{tabular}

TABLE I

SERVICE TYPE PARAMETERS

\section{A. Scenario 1}

The network-based decision was implementated. Initially, 400 mobile stations are randomly placed such that the
$80 \%$ of mobile stations generate voice traffic, and the $20 \%$ generate data traffic by randomly choosing service types. New mobile stations join the networks following the Poisson distribution with average arrival rate 20 mobile stations per minute during the entire simulation time. From time 0 to time 2,200 seconds, no station departs from the network, and the new mobile stations joined in this time period only generate voice traffic, hence, the network becomes gradually saturated with voice traffic. From time 2,200 to 3,500 seconds, mobile stations with voice traffic depart the network with average departure rate 20 stations per minute, while mobile stations with data traffic continue to arrive with average arrival rate 20 stations per minute. (No new station with voice traffic arrives from time 2,200 to 3,500 seconds.) Hence, the network becomes dominated by data traffic in this time period.

Figure 1(a) shows the throughput of each cell type (we only show the performance of a single cell in each type as cells of the same type perform similarly). The following observations are made from this simulation result, which conforms the operational policy rules. $\bullet$ During the almost entire simulation period, the CDMA1x cell fully serves for voice traffic with almost full load while the WCDMA part of the WCDMA-HSDPA cell serves for the voice traffic increasingly between simulation time 0 to 2,200 seconds and decreasingly between simulation time 2,200 to 3,500 seconds.

- During the first 1,200 seconds, the HSDPA cell (i.e., the HSDPA part of the WCDMA-HSDPA cell) has higher throughput than EvDO cell. This is because the network is not yet fully saturated with the voice traffic, and in this time period, the price of the HSDPA cell is lower than the price of the EvDO cell due to the higher capacity of the HSDPA cell.

- The throughput of HSDPA cell decreases from simulation time around 500 seconds to 2,200 seconds as WCDMA increasingly take more voice calls, and it increases from simulation time 2,200 through the end as the voice traffic taken by the WCDMA decreases.

Figure 1(b) shows the weighted call drop with and without our CRRM applied in which the weighted call drop denotes the total amount bandwidth requested by the mobiles stations dropped from the network. The following observations are made from the results shown in this figure.

- When the CRRM is applied, in simulation time around 1,700 seconds, the voice input reaches $92.5 \%$ of the network's voice capacity and the voice call drop starts at around the same time.

- When the CRRM is applied, in simulation time around 2,100 seconds, the voice input reaches the voice capacity of the network with about $4 \%$ call drop.

- When the CRRM is applied, in simulation time around 2,400 seconds, the data input reaches $90.7 \%$ of the network's remaining capacity after serving voice traffic, the data call drop starts at around the same time.

- When the CRRM is not applied (i.e., no vertical handover is performed after each mobile station is admitted to one of the accessible cell), voice calls start to drop from simulation time around 500 seconds and continue to drop significantly. Data calls also drop significantly when compared to the data call drop with the CRRM applied. 


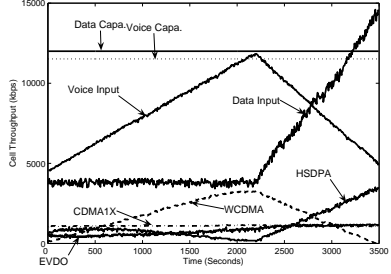

(a)

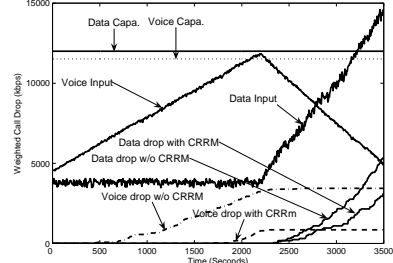

(b)

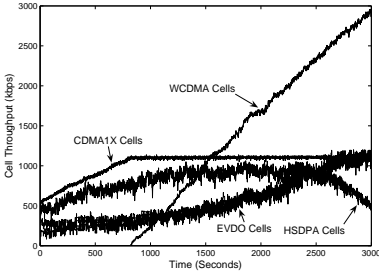

(c)

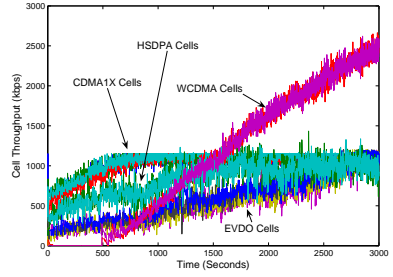

(d)

Fig. 1.

\section{B. Scenario 2}

Initially 200 mobile stations are randomly placed such that the $80 \%$ of them generate voice traffic, and the $20 \%$ generate data traffic randomly selecting service types from Table I. The simulation time is 3,000 seconds, and new mobile stations arrive in the area where all four types of RATs are deployed following the Poisson distribution with the average arrival rate 15 mobile stations per minute during the entire simulation period. The $80 \%$ of the new incoming mobile stations generate voice traffic while the remaining $20 \%$ generate data traffic. The utility value is updated in every second.

Figure 1(c) shows the throughput of each cell type when the network-based CRRM is applied while Figure 1(d) shows the throughput when the MS-based CRRM is applied. We observe here that the performance of each cell type when the MS-based CRRM is applied fluctuates a lot while it is rather stable when the network-based CRRM is applied.

\section{CONCLUSIONS AND Future WORK}

In this paper, we proposed a novel technique for access selection in multi-access networks. Our algorithms are based on utility values computed by marginal utility functions incorporating any given operational policy rules. Two possible implementations were discussed: network-based and MS-based implementations. Results obtained through simulations using the MANSim simulator that we developed showed that the network-based CRRM approach performs significantly better than the performance without CRRM or the MS-based approach. It should be interesting to further analyze the tradeoff in terms of the performance gain and the system overhead. Such an effort should be based within the standard activity such as the MIH protocol, and we plan to extend our research efforts to this direction.

\section{ACKNOWLEDGMENT}

This work was in part supported by the Access Network \& Mobile Terminal R \& D Center, SK Telecom, Seoul, Korea.

\section{REFERENCES}

[1] N. Niebert, M. Prytz, A. Schieder, N. Papadoglou, L. Eggert, F. Pittmann, and C. Prehofer, "Ambient networks: A framework for future wireless internetworking," in Proc. IEEE Vehicular Technology Conference - Spring, May 2005.
[2] F. Berggren, A. Bria, L. Badia, I. Karla, emco Litjens, P. Magnusson, F. Meago, H. Tang, and R. Veronesi, "Multi-radio resource management for ambient networks," in Proc. of IEEE 16th International Symposium on Personal, Indoor, and Mobile Radio Communications, Sep 2005.

[3] S. H. Y. W. L. S. M. L. S. M. Buddhikot, G. Chandranmenon, "Integration of 802.11 and third-generation wireless data networks," in IEEE INFOCOM, Mar 2003.

[4] H. H. Kalle Ahmavaara and R. Pichna, "Interworking architecture between $3 g p p$ and wlan systems," in IEEE Communications Magazine, Nov 2003.

[5] A. K. SALKINTZIS, "Interworking techniques and architectures for wlan/3g integration toward $4 \mathrm{~g}$ mobile data networks," in IEEE Wireless Communications, Jun 2004.

[6] H. S. Mahmood and B. Gage, "An architecture for integrating cdma2000 and 802.11 wlan networks," in Proc. IEEE Vehicular Technology Conference, Oct 2003.

[7] A. K. SALKINTZIS, "Interworking between wlans and thirdgeneration cellular data networks," in Proc. IEEE Vehicular Technology Conference, Apr 2003.

[8] M. Jaseemuddin, "An architecture for integrating umts and 802.11 wlan networks," in Eighth IEEE International Symposium on Computers and Communications, Jul 2003.

[9] A. K. SALKINTZIS, "Interworking techniques and architectures for wlan $/ 3 \mathrm{~g}$ integration toward $4 \mathrm{~g}$ mobile data networks," in IEEE Wireless Communications, Jun 2004.

[10] D. Kim and A. Ganz, "Architecture for 3g and 802.16 wireless networks integration with qos support," in International Conference on Quality of Service in Heterogeneous Wired/Wireless Networks (QShine 2005), 2005, p. 28.

[11] E. Brewer, "A network architecture for heterogeneous mobile computing," 1998. [Online]. Available: citeseer.ist.psu.edu/brewer98network.html

[12] B. Xing and N. Venkatasubramanian, "Multi-constraint dynamic access selection in always best connected networks," in MOBIQUITOUS '05: Proceedings of the The Second Annual International Conference on Mobile and Ubiquitous Systems: Networking and Services. Washington, DC, USA: IEEE Computer Society, 2005, pp. 56-64.

[13] D. Mariz, I. Cananea, D. Sadok, and G. Fodor, "Simulative analysis of access selection algorithms for multi-access networks," in WOWMOM '06: Proceedings of the 2006 International Symposium on on World of Wireless, Mobile and Multimedia Networks. Washington, DC, USA: IEEE Computer Society, 2006, pp. 219-227.

[14] O. Yilmaz, A. Furuskar, J. Pettersson, and A. Simonsson, "Access selection in wcdma and wlan multi-access networks," in Proc. IEEE Vehicular Technology Conference, May 2005.

[15] J. P. Romero, O. Sallent, R. Agusti, and M. A. Diaz-Guerra, Radio Resource Management Strategies in UMTS. WILEY, 2005.

[16] Draft IEEE Standard for Local and Metropolitan Area Networks: Media Independent Handover Services, "IEEE P802.21/S01.00," Tech. Rep., 2006. 